DE LA FUENTE, Hanns; MEJÍAS, Claudia; CASTRO, Pía. “Análisis econométrico de los determinantes de la criminalidad en Chile."

Polít. crim. Vol. 6, No 11 (Julio 2011), Doc. 1, pp. 192 - 208.

[http://www.politicacriminal.cl/Vol_06/n_11/Vol6N11D1.pdf]

\title{
Análisis econométrico de los determinantes de la criminalidad en Chile
}

\author{
Hanns de la Fuente Mella \\ Doctor, Profesor Asociado, Escuela de Comercio, \\ Facultad de Ciencias Económicas y Administrativas, \\ Pontificia Universidad Católica de Valparaíso. \\ Departamento de Modelación y Gestión Industrial, \\ Facultad de Ingeniería, Universidad de Talca. \\ hanns.delafuente@ucv.cl \\ Claudia Mejías Navarro \\ Ingeniero Civil Industrial, Universidad de Talca \\ claud_mejias@hotmail.com \\ Pía Castro O’Kuinghttons \\ Licenciado en Ciencias de la Ingeniería, Universidad de Talca \\ piacastro@alumnos.utalca.cl
}

\section{Resumen}

Los altos índices delictuales que presenta la sociedad chilena en los últimos años han generado una constante inquietud y preocupación tanto en el gobierno como en investigadores, aumentando así la importancia de las líneas de investigación que tratan de explicar los determinantes de la delincuencia en el país. El presente estudio se realiza por medio de formulaciones matemáticas y de su correspondiente especificación econométrica a través del uso de panel de datos, lo que permite diseñar modelos de análisis con los cuales determinar los factores que motivan a una persona a delinquir. Los resultados del estudio arrojan que la criminalidad está determinada por diversos factores socioeconómicos, demográficos, disuasorios y de políticas públicas, los cuales pueden afectar de manera positiva o negativa en la criminalidad.

\section{Palabras Clave:}

Modelos econométricos, datos de panel, delincuencia.

\begin{abstract}
The high rates of criminality shown in Chilean society in recent years, have generated concern in both government and researchers, thus increasing the importance of lines of research trying to explain the variables that influence crime rates in the country. The following research was conducted through mathematical formulations and the corresponding econometric specification through the use of panel data, allowing for the design of analysis models with which the factors that motivate a person to commit a crime can be determined. The study results show that
\end{abstract}


DE LA FUENTE, Hanns; MEJÍAS, Claudia; CASTRO, Pía. “Análisis econométrico de los determinantes de la criminalidad en Chile."

criminality is determined by many factors such as: socioeconomic, demographic, dissuasive and of public policies, that may affect criminality positively or negatively.

\section{Keywords:}

Econometrics models, data panel, delinquency.

\section{Introducción}

Los índices de criminalidad y la sensación de inseguridad de la población son factores que atentan contra la calidad de vida de las personas, así, en el mundo y específicamente en Chile, hay una constante preocupación de las autoridades por conocer los factores que conducen a los individuos a cometer un delito.

Las razones que motivan este estudio son comprender los factores que motivan a los individuos a delinquir, objeto presentar las principales relaciones entre dichas variables y su grado de influencia, así, las autoridades tendrán una herramienta que les permitirá tomar decisiones basadas en criterios cuantitativos, y con esto proponer medidas para obtener mejoras en el combate contra la delincuencia. Para esto, se identifican cuáles son los factores determinantes de la delincuencia, con ellos se crea una base de datos que comprende desde el año 1990 a 2008, y se proponen modelos tentativos que se evalúan y validan con el fin de construir un modelo econométrico definitivo.

La criminología tradicionalmente ha sido investigada desde el punto de vista psicológico, sin embargo, han tomado gran importancia las investigaciones que incluyen aspectos sociales y económicos, entre las variables que ejercen influencia en las tasas del delito. El análisis económico del delito se origina de la idea utilitarista del individuo de los economistas neoclásicos y de sus razonamientos acerca del individualismo, el comportamiento maximizador del beneficio, las preferencias estables y el equilibrio del mercado.

En la literatura existen dos enfoques teóricos principales para estudiar los determinantes de la criminalidad. La primera perspectiva es fundada por sociólogos, psicólogos, criminólogos y cientistas políticos quienes postulan, que la participación de los individuos en las actividades ilegales corresponde a un comportamiento poco racional, siendo producto de la predisposición de una persona hacia el crimen, la cual puede basarse tanto en el temperamento de un individuo como en su entorno social. Por otra parte, existe el pensamiento conocido como "la teoría económica del crimen", creada por Becker ${ }^{1}$ y Ehrlich, ${ }^{2}$ cuyo razonamiento está orientado a determinantes del tipo económico, las cuales se dividen en dos tipos, la teoría microeconómica y macroeconómica del crimen, este último será el enfoque de la presente investigación, donde a través del uso de modelos econométricos, será posible determinar los efectos de cada uno de los determinantes de la delincuencia sobre los delitos de mayor connotación social.

\footnotetext{
${ }^{1}$ BECKER Gary, "Crime and Punishment: An Economic Approach", Journal of Political Economy Vol. 76, N ${ }^{\circ}$ (1986).

${ }^{2}$ EHRLICH I., "Participation in Illegitimate Activities: A Theoretical and Empirical Investigation", Journal of Political Economy Vol. 81, Nº 3 (1973).
} 
Polít. crim. Vol. 6, № 11 (Julio 2011), Doc. 1, pp. 192 - 208.

[http://www.politicacriminal.cl/Vol_06/n_11/Vol6N11D1.pdf]

\section{Materiales y Métodos}

\subsection{Marco Teórico}

La criminología tradicionalmente ha sido investigada desde el punto de vista psicológico. Sin embargo, han tomado gran importancia las investigaciones que incluyen aspectos sociales y los económicos entre las variables que ejercen influencia en las tasas del delito. El análisis económico del delito se origina de la idea utilitarista del individuo, de los economistas neoclásicos y de sus razonamientos acerca del individualismo, el comportamiento maximizador del beneficio, las preferencias estables y el equilibrio del mercado.

En el análisis económico, las investigaciones sobre el delito se han orientado casi exclusivamente en sus determinantes y/o consecuencias de tipo económico. Basándonos en esta orientación, podemos encontrar dos ramas dominantes en la literatura. La primera rama es el enfoque microeconómico, el cual pretende explicar el delito como consecuencia de una elección racional a nivel personal, es decir, los sujetos que delinquen lo hacen en función de un análisis costo/beneficio y se ha pretendido demostrar la teoría de la disuasión a partir de diversos análisis. La segunda rama corresponde al enfoque macroeconómico, el cual se centra en investigar la relación que existe entre el delito como variable global y otras variables agregadas.

La teoría económica del crimen planteada por Becker ${ }^{3}$ y Ehrlich, ${ }^{4}$ plantea que la criminalidad debe examinarse a partir de los incentivos económicos que los individuos enfrentan para delinquir, y concibe la participación en actividades ilegales como el producto de un proceso de maximización de utilidades de individuos racionales bajo incertidumbre.

En otras palabras, en la teoría económica del delito y el castigo se parte presumiendo la racionalidad del sujeto. Éste se plantea la posibilidad de cometer un delito, evaluando los beneficios que puede obtener al violar la ley, como también considera y evalúa los riesgos que el acto criminal implica. Tras efectuar un análisis de costo/beneficio, sólo en el caso de que los beneficios compensen considerablemente los costos y riesgos, posiblemente el individuo realice el delito, siempre y cuando sus principios morales no sean un elemento de disuasión. Por lo tanto, los delincuentes no son observados, en principio, como sujetos desviados, sino como personas normales, como agentes maximizadores que evalúan el costo y el beneficio de su actividad, y el castigo sería el precio, resultado del equilibrio entre demanda de seguridad y oferta de delito. El sistema judicial sería un mercado que podría alcanzar la combinación deseada entre seguridad y gasto.

Desde el año 2000 en Chile, rige un nuevo sistema de administración de justicia penal, que pretende reemplazar por completo al que existe en nuestro país desde el siglo pasado. Esta reforma es el nuevo Código Procesal Penal, publicado el 12 de octubre del 2000, que sustituye el antiguo sistema inquisitivo por uno acusatorio. La idea es que esta nueva justicia siga los modelos existentes en países desarrollados, donde la administración de justicia penal es más eficiente, los acusados y víctimas de delitos cuentan con derechos explícitos y las causas no se acumulan por la propia naturaleza de su conducción.

\footnotetext{
${ }^{3}$ BECKER, Crime and Punishment, cit. nota ${ }^{\circ} 1$, p. 2.

${ }^{4}$ EHRLICH, Participation in Illegitimate Activities, cit. nota ${ }^{\circ} 2$, p. 2.
} 
DE LA FUENTE, Hanns; MEJÍAS, Claudia; CASTRO, Pía. “Análisis econométrico de los determinantes de la criminalidad en Chile."

Respecto a los determinantes de la delincuencia, para la presente investigación los clasificaremos en los siguientes factores: socioeconómicos, demográficos, disuasorios y políticas públicas.

\subsubsection{Factores Socioeconómicos}

La pobreza, el desempleo, la desigualdad de ingresos, el nivel de escolaridad y la cantidad de inmigrantes ilegales de un país, habitualmente se indican como importantes causales de la delincuencia, no obstante, la evidencia empírica es escasa. La pobreza, según describen Gutiérrez, Nuñez y Rivera, ${ }^{5}$ se determina en el momento que un individuo no logra adquirir bienes básicos para vivir. Además, los índices de desempleo interactúan de manera muy cercana con los de delincuencia Machin y Meghir, ${ }^{6}$ Beyer y Vergara, ${ }^{7}$ Ruiz et al.. ${ }^{8}$ Otro factor influyente en los índices de delincuencia, es la desigualdad de ingresos que se presenta en el país, cuya teoría corresponde a Bandrés, ${ }^{9}$ argumentando que para reducir el nivel de delincuencia se debe reducir la desigualdad en el reparto de la renta. Adicionalmente un factor social considerado en esta investigación, es el nivel de educación, el cual, es destacado por diversos autores (Lochner y Moretti, ${ }^{10}$ Buonanno, ${ }^{11}{ }^{12}$ y Buonanno/Leonida ${ }^{13}$ ), ya que la educación constituye un indicador de la posible generación de ingresos procedentes de actividades legales. La inmigración ilegal está ligada al nivel de delincuencia si se analiza de un punto de vista económico, ya que general las personas inmigrantes que no cuentan con estadía legalizada, se asocian a la pobreza, dado que habitualmente vienen al país en busca de mejores oportunidades laborales.

\subsubsection{Factores Demográficos}

Dentro de los factores demográficos estudiados por la literatura, encontramos al género, a la tasa de urbanidad, a la edad de los potenciales delincuentes, a la tasa de las mujeres jefas de hogar y al consumo de drogas. Así, de acuerdo a lo señalado por Ruiz et al., ${ }^{14}$ la mayoría de los delincuentes son hombres, señalando en su estudio que el género de las personas corresponde a uno de los principales predictores del crimen. Por su parte, de acuerdo a la investigación desarrollada por Wilson y Herrenstein, ${ }^{15}$ los delitos en su mayoría son realizados por hombres. En general se

${ }^{5}$ GUTIÉRREZ M., NÚÑEZ J. y RIVERA J., "Caracterización socioeconómica y espacial de la criminalidad en Chile", Revista Cepal № 98 (2008), pp. 165-180.

${ }^{6}$ MACHIN S., y MEGHIR C., "Crime and Economic Incentives", Journal of Human Resources, Vol. 39 N4 (2004), pp. 958-979.

BEYER H., y VERGARA R., Delincuencia en Chile: Determinantes y Rol de las Políticas Públicas, mimeo., Pontificia Universidad Católica de Chile, 2006.

${ }^{8}$ RUIZ P., CEA M., RODRÍGUEZ C., MATUS J.P., "Determinantes de la criminalidad: Análisis de Resultados.", Política Criminal No3 (2007), pp. 1-80.

${ }^{9}$ BANDRÉS E. y DÍEZ-TICIO A., "Delincuencia y acción policial: Un enfoque económico", Revista de Economía Aplicada, Vol. IX, No27 (2001), p. 5-33.

${ }^{10}$ LOCHNER L., y MORETTI E., The Effect of Education on Crime: Evidence from Prison Inmates, Arrests, and Self-Reports, NBER Working paper $\mathrm{N}^{\circ} 8605,2001$.

${ }^{11}$ BUONANNO P., Crime, education and peer pressure, Working paper $\mathrm{N}^{\circ} 64$, Milán: Università degli Studi di Milano, 2003.

${ }^{12}$ BUONANNO P., Identifying the effect of education on crime. Evidence from the Italian Regions, Working paper $N^{\circ}$ 55, Milán: Università degli Studi di Milano, 2003.

${ }^{13}$ BUONANNO P. y LEONIDA L., Criminal activity and education: evidence from Italian Regions, Working paper $\mathrm{N}^{\circ} 3$, Bergamo: Università degli Studi di Bergamo, 2005.

${ }^{14}$ RUIZ/CEA/RODRÍGUEZ/MATUS, Determinantes de la criminalidad, cit. nota $\mathrm{n}^{\circ} 8$, p. 3.

${ }^{15}$ WILSON J. y HERRENSTEIN R., Crime and Human Nature: The Definitive Study of the Causes of Crime, New York: Simon \& Schuster, 1985. 
Polít. crim. Vol. 6, № 11 (Julio 2011), Doc. 1, pp. 192 - 208.

[http://www.politicacriminal.cl/Vol_06/n_11/Vol6N11D1.pdf]

espera que las conductas delictivas sean mayores en zonas con una mayor densidad de población (Beyer y Vergara, ${ }^{16}$ Rodríguez $^{17}$ ). Por ejemplo, en áreas con una alta densidad de población, la probabilidad de ser capturado es menor, induciendo a los individuos a cometer delitos, por lo que la densidad urbana es una variable fundamental en la explicación delictiva. También se encuentra evidencia que muestra que los jóvenes son más proclives a participar en actividades delictivas, produciéndose un alza durante la adolescencia alcanzando su peak antes de los 20 años y luego reduciéndose gradualmente Blumenstein et al., ${ }^{18}$ Marvell y Moody, ${ }^{19}$ Beyer y Vergara. ${ }^{20}$ De acuerdo a lo descrito por Glaeser et al., ${ }^{21}$ quienes indican que los individuos que han crecido en un hogar donde una mujer sea la jefa de éste, poseen tanto un deficiente desarrollo de las habilidades no cognitivas, como desenvolvimiento social, sin embargo, un estudio realizado a la población chilena por Beyer y Vergara, ${ }^{22}$ se observa que un bajo desarrollo cognitivo y social en los jóvenes, no depende precisamente de que el jefe de su hogar sea una mujer. Por otra parte, Ruiz et al. ${ }^{23}$ mencionan que cuando aumenta el consumo de droga aumenta el delito, lo cual se debe a los efectos que produce la ingesta de dichas substancias, la búsqueda y medios para conseguirlas, además de disputas entre micro y macro traficantes, entre otros factores.

\subsubsection{Factores Disuasorios.}

La economía del delito establecida por $\operatorname{Becker}^{24}$ y posteriormente ampliada por Ehrlich, ${ }^{25}$ en la cual se asume que los criminales se comportan racionalmente, y por esta razón, responden tanto a incentivos y castigos, como costos y beneficios de delinquir, implica que los factores que influyen al momento de decidir cometer un delito son por ejemplo: la presencia y efectividad policial, la dureza de las penas y, la probabilidad de ir a la cárcel. Por una parte, Levitt, ${ }^{26}$ Corman y Mocan ${ }^{27}$ han demostrado experimentalmente que la mayor presencia policial reduce los niveles de criminalidad en una sociedad, por otra Marvell y Moody ${ }^{28}$ y Levitt $^{29}$ lo han demostrado respecto a las mayores tasas de encarcelación.

\footnotetext{
${ }^{16}$ BEYER/VERGARA, Delincuencia en Chile, cit. nota $\mathrm{n}^{\circ}$ 7, p. 3.

${ }^{17}$ RODRÍGUEZ A., "Los determinantes socio-económicos del delito en España.", Revista Española de Investigación Criminológica $\mathrm{N}^{\circ} 1$ (2003).

${ }^{18}$ BLUMENSTEIN A., COHEN J., ROTH J. y VISHER E., Criminal Careers and "Career Criminals", Washington DC: National Academy of Sciences; Santiago: Departamento de Economía Universidad de Chile, 1986.

${ }_{19}$ MARVELL T., y MOODY, C., "Age Structure and Crime Rates: The Conflicting Evidence", Journal of Quantitative Criminology, Vol. 7, $\mathrm{N}^{\circ} 3$ (1991), pp. 237-273.

${ }^{20}$ BEYER/ VERGARA, Delincuencia en Chile, cit. nota ${ }^{\circ}$ 7, p. 3.

${ }^{21}$ GLAESER E., SACERDOTE B., y SCHEINKMAN J., "Crime and Social Interactions", Quarterly Journal of Economics Vol. 111 (1996), pp. 507-548.

${ }^{22}$ BEYER/VERGARA, Delincuencia en Chile, cit. nota $\mathrm{n}^{\circ}$ 7, p. 3.

${ }^{23}$ RUIZ/CEA/RODRÍGUEZ/MATUS, Determinantes de la criminalidad, cit. nota ${ }^{\circ}$ 8, p. 3.

${ }^{24}$ BECKER, Crime and Punishment, cit. nota ${ }^{\circ} 1$, p. 2.

${ }^{25}$ EHRLICH, Participation in Illegitimate Activities, cit. nota $\mathrm{n}^{\circ}$ 2, p. 2.

${ }^{26}$ LEVITT S., "Using electoral cycles in Police Hiring to Estimate the Effect of Police on Crime", American Economic Review, Vol. 87, № 3 (1997).

${ }^{27}$ CORMAN H. y MOCAN H., "A Time-Series Analysis of Burglary, Deterrence, and Drug Abuse in New York City", American Economic Review Vol. 90 (2000), pp. 584-604.

${ }^{28}$ MARVELL T. y MOODY C., "Prison population growth and crime reduction", Journal of Quantitative Criminology, Vol. 10, No 1 (1994), pp. 109-140.

${ }^{29}$ LEVITT S., "The Effect of Prison Population Size on Crime Rates: Evidence from Prison Overcrowding Litigation", Quarterly Journal of Economics Vol. CXI (1996), pp. 319-352.
} 
DE LA FUENTE, Hanns; MEJÍAS, Claudia; CASTRO, Pía. “Análisis econométrico de los determinantes de la criminalidad en Chile."

\subsubsection{Factores asociados a las Políticas Públicas.}

Como ya se ha mencionado, en el año 2000 se ha puesto en Chile en marcha la Reforma Procesal Penal (RPP), la cual se aplica a todo el país y su principal objetivo es modernizar el código de procedimiento penal, vigente desde 1906, adaptándose al modelo de países desarrollados más eficientes en las condenas. Asimismo, en los últimos años ha nacido la iniciativa del gobierno para reforzar la seguridad ciudadana, a través del llamado "Plan Cuadrante y Comuna Segura", con el objetivo de lograr una comunidad más segura y unida.

\subsection{Análisis de Datos}

El estudio utiliza una base de datos para las 13 regiones de Chile, las cuales comprenden el periodo 1990 a 2008 debido a que en este periodo es donde se disponen series de las variables relevantes para el análisis. La variable a explicar en esta investigación será el nivel de delincuencia por regiones, utilizando para ello la proxy tasa de denuncias de los delitos. Se utiliza la tasa de denuncias debido a que en Chile existen estos datos para el periodo de tiempo de estudio, así, en este estudio la variable dependiente será la tasa de denuncias por cada cien mil habitantes.

\subsubsection{Variable Dependiente}

La variable dependiente de esta investigación será la delincuencia, utilizando la proxy tasa de denuncias, para la selección de esta variable es importante considerar que los delitos no tienen la misma probabilidad de ser denunciados, además, el cambio en la legislación chilena puede alterar las mediciones de las denuncias $y$, entonces, la tasa de denuncias totales no se considera una buena aproximación de la delincuencia en Chile, sin embargo, se selecciona esta por la disponibilidad de la data al momento de la realización del estudio. Así, la variable dependiente que se seleccionará en esta investigación es la tasa de denuncias por robo con fuerza por cada 100.000 habitantes. ${ }^{30}$

Para la medición de la variable dependiente, se incluirán en este estudio variables de control, las cuales nos permiten medir el comportamiento de la variable dependiente, estás variables de control serán: robo con fuerza, robo con violencia y hurto. Las tres variables mencionadas son tomadas del Anuario de Estadísticas de Carabineros y los indicadores para medir la variable dependiente, serán:

- Tasa de denuncia por robo con fuerza en Chile para un año X (cada 100 mil habitantes).

- Tasa de denuncia por robo con violencia en Chile para un año X (cada 100 mil habitantes).

- $\quad$ Tasa de denuncia por hurto en Chile para un año X (cada 100 mil habitantes).

\footnotetext{
${ }^{30}$ Cada 100.000 habitantes corresponde al estándar internacional que se usa para presentar las estadísticas de delitos con el fin de corregir las cifras por el efecto de la población.
} 
Polít. crim. Vol. 6, No 11 (Julio 2011), Doc. 1, pp. 192 - 208.

[http://www.politicacriminal.cl/Vol_06/n_11/Vol6N11D1.pdf]

\subsubsection{Variables Explicativas}

Las variables explicativas que se seleccionan son agrupadas en socioeconómicas, demográficas, disuasorias y políticas públicas. Los datos son extraídos del Ministerio de Cooperación y Planificación (MIDEPLAN), mayoritariamente de la Encuesta de Caracterización Socioeconómica Nacional (CASEN), ${ }^{31}$ Instituto Nacional de Estadísticas (INE), ${ }^{32}$ Banco Central, ${ }^{33}$ Ministerio del Interior ${ }^{34}$ y el Consejo Nacional para el Control de estupefacientes (CONACE) ${ }^{35}$ Las variables relacionadas con el costo de delinquir son extraídas de Anuarios de Estadísticas de Carabineros, ${ }^{36}$ y los factores relacionados con los planes anti delincuencia implementados en los últimos años son recopilados directamente de Carabineros de Chile. A continuación se especifica cada variable usada en este estudio.

\section{a) Variables Socioeconómicas}

- Pobreza: Se entiende como pobreza a la condición que puede padecer un individuo, que estará determinada, esencialmente por su ingreso monetario, que no permite adquirir los bienes básicos para vivir. En Chile, los individuos que tengan ingresos inferiores a una canasta básica, serán llamados "pobres indigentes", y quienes tengan ingresos inferiores a dos canastas y superiores a una, serán llamados "pobres no indigentes".

- Desempleo: Un individuo se encuentra en la condición de desempleado, si estando en la población económicamente activa, no tiene empleo, pero lo sigue buscando.

- Desigualdad: La desigualdad corresponde a la carencia relativa de las personas entre sí en un lugar y tiempo determinado. Para fines de este estudio, se conceptualizará la desigualdad como el estado que vive una nación cuando ésta tiene diferencias significativas de ingreso entre distintos grupos de habitantes.

- Nivel de Escolaridad: Se refiere al capital humano con que cuenta un individuo, lo que sería un reflejo de las oportunidades, principalmente laborales, con las que dispondría. Los años de escolaridad son la cantidad de años que aprueba un individuo en el sistema escolar.

- Inmigración: Se refiere a las personas que vienen de un país extranjero a vivir al propio. El indicador que requiere el estudio son los inmigrantes ilegales, sin embargo, el Departamento de Extranjería y Migración carece de este dato. Pero según Ruiz et. al., ${ }^{37}$ en su estudio de criminalidad, indican que el dato de los regulares sirve como indicador de la tendencia de los irregulares.

\section{b) Variables Demográficas}

- Género: Corresponde al sexo de los potenciales delincuentes.

- Tasa de Urbanidad: Corresponde a la relación entre la cantidad de personas que habitan en zonas urbanas con respecto a la población total.

\footnotetext{
${ }^{31}$ Encuesta Casen desde 1990 a 2006. http://www.mideplan.cl

32 http://www.ine.cl

33 http://www.bcentral.cl

34 http://www.interior.gov.cl

35 http://www.conace.cl

${ }^{36}$ Fuentes: Fundación Paz Ciudadana e Instituto Nacional de Estadística.

${ }^{37}$ RUIZ/CEA/RODRÍGUEZ/MATUS, Determinantes de la criminalidad, cit. nota n ${ }^{\circ}$ 8, p. 3.
} 
DE LA FUENTE, Hanns; MEJÍAS, Claudia; CASTRO, Pía. “Análisis econométrico de los determinantes de la criminalidad en Chile."

- Etariedad: La estructura etaria corresponde a un factor que caracteriza a la población total del país o región según la distribución de las edades de sus componentes.

- Mujeres Jefas de Hogar: Número de hogares donde el jefe de hogar corresponde al sexo femenino.

- Drogas: Se consideró para esta investigación, el consumo de drogas con más incidencia a nivel de los datos, es decir, las que más se consumen.

c) Variables de Disuasión

- Aprendidos Robo con Fuerza: Los aprehendidos son aquellas personas a quienes se ha privado de libertad en forma transitoria por considerarse sospechoso de cometer un delito o por creer que no prestarán la cooperación oportuna a que los obliga la ley en la investigación de un delito. Esta variable está principalmente relacionada con la Teoría Económica del Delito, de Becker ${ }^{38}$ y Ehrlich. ${ }^{39}$

- Eficacia Policial: La eficacia policial es entendida como la capacidad de la policía de obtener el resultado esperado que consiste en el arresto del supuesto delincuente. Esta variable consiste en la relación de los arrestos y denuncias en un tiempo t.

\section{d) Variables Relacionadas con Políticas Públicas}

En esta etapa se pueden considerar tres tipos de variables como Plan Cuadrante, Comuna Segura y Reforma Procesal penal. Sin embargo, para este estudio se analiza sólo la primera de ellas debido a que no se encuentra la información en el tiempo de realización de la investigación. Es bueno aclarar que en base a la metodología de esta investigación, el tiempo transcurrido desde que dicha reforma se comienza a implementar es demasiado breve ya que se implementa completamente el año 2005.

- Plan Cuadrante de Carabineros: Sistema de vigilancia preventiva permanente durante las 24 horas del día, que delimita un territorio cuya vigilancia es asignada a una dotación específica de policías encargados de velar por la seguridad ciudadana. El Plan Cuadrante se introdujo de forma experimental el año 1998 con un piloto en la Prefectura Santiago Sur, desde este año se fueron incorporando el resto de las regiones del país.

\subsection{Formulación de los Modelos}

Las variables endógenas de los modelos que se consideran en este estudio serán: Robo con Fuerza, Robo con Violencia, y Hurto, es decir, se estudiarán tres modelos para las 13 regiones del país, para el periodo 1990 a 2008, donde las secciones transversales serán las cada una de las regiones y el periodo corresponderá a la dimensión temporal, configurando así el panel de datos.

El modelo general de regresión de datos de panel para este estudio se especifica de la siguiente forma:

\footnotetext{
${ }^{38}$ BECKER, Crime and Punishment, cit. nota $\mathrm{n}^{\circ} 1$, p. 2.

${ }^{39}$ EHRLICH, Participation in Illegitimate Activities, cit. nota ${ }^{\circ} 2$, p. 2.
} 
Polít. crim. Vol. 6, No 11 (Julio 2011), Doc. 1, pp. 192 - 208.

[http://www.politicacriminal.cl/Vol_06/n_11/Vol6N11D1.pdf]

$$
Y_{i t}=\alpha_{i t}+X_{i t} \beta+\mu_{i t} \quad \text { (1) } \quad \text { con } \quad i=1,2, \ldots, N \quad t=1990,1992, \ldots, T
$$

donde $i$ se refiere a cada región del país (corte transversal), $t$ se refiere a la dimensión en el tiempo (1990-2008), $\alpha$ es un vector de interceptos de $\mathrm{n}$ parámetros, $\beta$ es un vector de $\mathrm{K}$ parámetros y $X_{i t}$ es la i-ésima observación al momento $t$ para las $\mathrm{K}$ variables explicativas.

En el caso de estudio, la muestra total de las observaciones en el modelo viene dado por $N \times T$, es decir, $13 \times 19=247^{40}$ observaciones.

Es relevante mencionar que existen dos procedimientos adicionales para estimar el modelo en un sistema de datos de panel. Uno de ellos implica el reconocimiento de que las variables omitidas pueden generar cambios en los interceptos ya sea a través del tiempo o entre unidades de corte transversal, en este caso el modelo de efectos fijos trata de aproximar estos cambios con variables dummy; el otro modelo es el de efectos aleatorios, que trata de capturar estas diferencias a través del componente aleatorio del modelo. Estas diferencias, es decir, la heterogeneidad propia de los componentes del panel, incluyen efectos observables como inobservables Greene. ${ }^{41}$ De acuerdo a esto, y con el fin de incorporar la heterogeneidad no observada, se explican los datos con el modelo de efectos fijos que considera que existe un término constante diferente para cada región, y supone que los efectos individuales son independientes entre sí. Además con este modelo se considera que las variables explicativas afectan por igual a las unidades de corte transversal y que estas se diferencian por características propias de cada una de ellas, medidas por medio del intercepto. Otro punto importante en la formulación de efectos fijos, es que las diferencias se incorporan a la constante del modelo Greene. ${ }^{42}$

Al aplicar efectos fijos de datos de panel, es interesante averiguar si los coeficientes del modelo de regresión, si bien son los mismos para todas las unidades de corte transversal (regiones) en un período dado, son distintos para período de tiempo diferentes (efecto fijo tiempo). Para el caso de un panel de datos con componente temporal dominante, importa averiguar si los coeficientes del modelo de regresión son distintos para cada unidad de corte transversal (región) cada variable explicativa pero constante a través del tiempo (efecto fijo producto).

El modelo de efectos fijos parte del supuesto de que los coeficientes varían dependiendo el producto o del momento del tiempo. En el caso de datos de panel cuya componente transversal es la dominante, es decir, existen más secciones transversales que períodos de tiempo, este modelo puede captar la variación existente en la muestra debido a la presencia de diferentes productos con la inclusión de una serie de variables dicotómicas $p_{i}$, una para cada producto. Se hace lo mismo para el caso de diferencias a través del tiempo, mediante la inclusión de un conjunto de variables dicotómicas $t_{t}$, una para cada momento del tiempo, Perez (2006). ${ }^{43}$

De acuerdo a lo mencionado, se debe diferenciar entre el tipo de efecto fijo que mejor incorpore esta heterogeneidad no observable, para esto, se evaluarán los dos efectos propuestos por la literatura. Por lo tanto, se estimarán los modelos de efectos fijos de tiempo y efectos fijos de

\footnotetext{
${ }^{40} \mathrm{~N}=13$ regiones del país, mapa político antiguo; $\mathrm{T}=19$ variables explicativas.

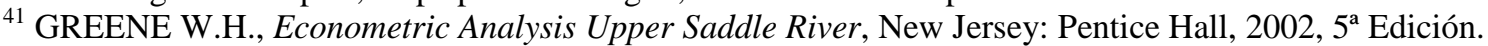

${ }^{42}$ GREENE, Econometric Analysis Upper Saddle River, cit. nota ${ }^{\circ} 41$.

${ }^{43}$ PÉREZ C., Econometría de las series temporales, Pearson- Prentice Hall.
} 
DE LA FUENTE, Hanns; MEJÍAS, Claudia; CASTRO, Pía. “Análisis econométrico de los determinantes de la criminalidad en Chile."

región, los que serán probados con el modelo de efecto común, o sea, se contrastarán con el modelo que considera para todos las regiones un término constante fijo.

Según Greene, ${ }^{44}$ para distinguir entre el modelo de efectos fijos que mejor represente la heterogeneidad se debe aplicar el estadístico de prueba F, la cual compara entre el modelo de efectos comunes y los posibles efectos fijos de datos de panel. El cálculo para este estadístico es el siguiente:

$$
F=\frac{\left(R_{E F}^{2}-R_{C M}^{2}\right) /(n-1)}{\left(1-R_{E F}^{2}\right) /(n * t-n-k)}
$$

Donde $R_{E F}^{2}$ y $R_{C M}^{2}$ corresponden a los coeficientes de determinación $\left(R^{2}\right)$ del modelo de efectos fijos y de efectos comunes respectivamente, $n$ corresponde al número de secciones transversales, $t$ al número de períodos de tiempo y $k$ al número de parámetros estimados.

La hipótesis que se usará para contrastar efectos fijos de tiempo y de producto con efectos comunes, se indica a continuación:

$$
\begin{aligned}
& H_{0}: \text { Efecto común mejor que efecto fijo. } \\
& H_{A}: \text { Efecto fijo mejor que efecto común. }
\end{aligned}
$$

De acuerdo a la metodología descrita, se presenta en la tabla 1 los resultados obtenidos al aplicar

\begin{tabular}{|c|c|c|c|c|c|c|}
\hline Modelo & \multicolumn{3}{|c|}{ Común v/s Fijo Tiempo } & \multicolumn{3}{|c|}{ Común v/s Fijo Producto } \\
\hline Delincuencia & $\mathbf{F}$ & $\begin{array}{l}\text { Valor }{ }^{45} \\
\text { Crítico }\end{array}$ & Conclusión & $\mathbf{F}$ & $\begin{array}{c}\text { Valor } \\
\text { Crítico }\end{array}$ & Conclusión \\
\hline
\end{tabular}
la prueba F para los tres modelos del estudio.

\begin{tabular}{|c|c|c|c|c|c|c|}
\hline $\begin{array}{l}\text { Robo con } \\
\text { Fuerza }\end{array}$ & 2,32 & 1,75 & $\begin{array}{l}\text { Fijo tiempo } \\
\text { mejor que } \\
\text { común }\end{array}$ & 62,27 & 1,75 & $\begin{array}{l}\text { Fijo producto } \\
\text { mejor que } \\
\text { común }\end{array}$ \\
\hline $\begin{array}{l}\text { Robo con } \\
\text { Violencia }\end{array}$ & 4,29 & 1,75 & $\begin{array}{l}\text { Fijo tiempo } \\
\text { mejor que } \\
\text { común }\end{array}$ & 48,71 & 1,75 & $\begin{array}{l}\text { Fijo producto } \\
\text { mejor que } \\
\text { común }\end{array}$ \\
\hline
\end{tabular}

Tabla 1: Resultados Prueba F para la comparación entre efectos fijos y comunes.

\footnotetext{
${ }^{44}$ GREENE, Econometric Analysis Upper Saddle River, cit. nota ${ }^{\circ} 41$.

${ }^{45}$ El valor crítico se extrajo de la tabla F de Fisher con $n-1$ grados de libertad del numerador y $n * t-k-n$ grados de libertad del denominador al $95 \%$ de confiabilidad.
} 
Polít. crim. Vol. 6, N 11 (Julio 2011), Doc. 1, pp. 192 - 208.

[http://www.politicacriminal.cl/Vol_06/n_11/Vol6N11D1.pdf]

\begin{tabular}{llllll}
\hline Hurto & 14,86 1,75 & $\begin{array}{l}\text { Fijo tiempo } \\
\text { mejor que } \\
\text { común }\end{array}$ & 847,02 & 1,75 & $\begin{array}{l}\text { Fijo producto } \\
\text { mejor que } \\
\text { común }\end{array}$ \\
\hline
\end{tabular}

Fuente: Elaboración Propia

Según los resultados obtenidos al contrastar la hipótesis de la prueba $F$, se constituyen a continuación las conclusiones respecto del efecto que mejor representa la heterogeneidad no observable entre las unidades de corte transversal, es decir, entre las regiones que constituyen el país. Para los modelos delictivos la selección se realiza en base a la significatividad individual y conjunta de los modelos estimados. De acuerdo a lo mencionado y analizado en los modelos tenemos que, para el modelo Robo con Fuerza el mejor efecto es el efecto fijo tiempo. Lo mismo ocurre para el modelo Robo con Violencia donde se selecciona aplicando la misma metodología, que el mejor efecto es el efecto fijo tiempo. En el caso del modelo Hurto se selecciona como mejor efecto al efecto fijo empresa, debido a la mejor significatividad de las variables presentadas en la estimación de este modelo.

\subsection{Resultados de la Estimación}

En base a la selección de los efectos desarrolladas en el apartado anterior, se continúa con la estimación de los tres modelos, para lo anterior se procede a eliminar datos atípicos y quitar variables no significativas en la estimación. Los resultados de las estimaciones se presentan a continuación. Los resultados de la estimación de los modelos de Robo con Fuerza y Robo con Violencia, considerando las trece regiones del país y un efecto fijo tiempo en ambos modelos, se presentan en las tablas 2 y 3 respectivamente.

Tabla 2: Resultados estimación Robo con Fuerza

\begin{tabular}{clllc}
\hline Variable & Coefficient & Std. Error & t-Statistic & Prob. \\
\hline EFICACIA_POLICIAL & -7.372388 & 0.202633 & -36.38297 & 0.0000 \\
HOMB_JOV & -88.17188 & 4.348764 & -20.27516 & 0.0000 \\
HOMB_JOV_POBRES & -8.026607 & 0.503021 & -15.95679 & 0.0000 \\
HOMB_JOV_URB_POBRES & 3.191236 & 0.561215 & 5.686302 & 0.0013 \\
HOMB_JOV_URB & 125.3436 & 5.612226 & 22.33403 & 0.0000 \\
PLAN_CUADRANTE & 1.800206 & 0.054840 & 32.82661 & 0.0000 \\
POBLACION_URB & -20.40590 & 0.719629 & -28.35616 & 0.0000 \\
APRE_ROBO_FUERZA & 3.312492 & 0.069194 & 49.29724 & 0.0000 \\
CONSUMO_DROGAS_MARIHUANA & 11.99508 & 0.650947 & 18.42713 & 0.0000 \\
DROGAS_APRE_C_100_MIL_HA & -1.186379 & 0.025515 & -46.49687 & 0.0000 \\
DROGAS_DEN_C_100_MIL_HA & 5.588120 & 0.620910 & 8.999888 & 0.0001 \\
EDAD_PROM_POBLACION & 7.636569 & 0.477018 & 16.00897 & 0.0000 \\
ESCOLARIDAD_PROM_JOV & 75.76536 & 6.064917 & 12.49240 & 0.0000 \\
ESCOLARIDAD_PROM_POBLACION & 76.25937 & 6.393299 & 11.92802 & 0.0000 \\
MUJ_JEFAS_HOGAR & 0.000901 & $1.37 \mathrm{E}-05$ & 65.75210 & 0.0000 \\
RAZON_DESIGUALDAD_NA & -237.3357 & 5.314289 & -44.65992 & 0.0000 \\
C & 139.9956 & 62.69348 & 2.233017 & 0.0670 \\
\hline
\end{tabular}


DE LA FUENTE, Hanns; MEJÍAS, Claudia; CASTRO, Pía. “Análisis econométrico de los determinantes de la criminalidad en Chile."

Como se observa en la tabla 2 la variable exógena analizada es Robo con Fuerza, al estimar el modelo con todas las variables explicativas es relevante mencionar que tres de ellas no presentaron significatividad, por lo cual fueron eliminadas del modelo. Las variables referidas corresponden a: razón desigualdad autónoma regional, línea de pobreza y desempleo. Las demás variables explicativas resultan ser significativas individualmente al 99\%. Referente a la significatividad conjunta, se observa que el modelo es significativo también al 99\%. Otro resultado importante de destacar es el coeficiente de determinación $\left(\mathrm{R}^{2}\right)$ que es de aproximadamente un 99,9\%. Respecto a la normalidad de los residuos según el estadístico de Jarque Bera, ${ }^{46}$ se obtiene un comportamiento normal de los datos. Finalmente se observa que la constante (C) no se elimina del modelo a pesar de resultar no significativa, ya que los efectos fijos de tiempo estimados, se ven reflejados en ésta. A continuación se presenta el modelo Robo con Fuerza en su forma linealizada considerando para ello el efecto fijo de tiempo:

$\mathrm{RF}_{\mathrm{it}}=139.9956-7.372388 *$ EFICIENCIA_POLICIAL $-88.17188 *$ HOMB_JOV 8.026607 $*$ HOMB_JOV_POBRES + $3.191236 *$ HOMB_JOV_URB_POBRES + $125.3436 *$ HOMB_JOV_URB + $1.800206 *$ PLAN_CUADRANTE $-20.40590 *$ POBLACION_URB + $3.312492 *$ APRE_ROBO_FUERZA + $11.99508 *$ CONSUMO_DROGAS_MARIHUANA $1.186379 *$ DROGAS_APRE_C_100_MIL_HA + 5.588120 $*$ DROGAS_DEN_C_100_MIL_HA + $7.636569 *$ EDAD_PROM_POBLACION + $75.76536 *$ ESCOLARIDAD_PROM_JOV + $76.25937 *$ ESCOLARIDAD_PROM_POBLACION + 0.000901 $*$ MUJ_JEFAS_HOGAR $237.3357 *$ RAZON_DESIGUALDAD_NA $+\mathrm{T}_{98} *(-23.59258)+\mathrm{T}_{00} *(-35.97127)+\mathrm{T}_{06} *$ (59.66385)

Donde: $T_{t}=1$ para el período $t(1990, \ldots, 2008)$ y $T_{t}=0$ en caso contrario;

$i=$ Regiones pertenecientes al mapa político antiguo chileno $(1, \ldots, 13)$.

Tabla 3: Resultados estimación Robo con Violencia

\begin{tabular}{ccccc}
\hline Variable & Coefficient & $\begin{array}{c}\text { Std. } \\
\text { Error }\end{array}$ & t-Statistic & Prob. \\
\hline DESEMPLEO & 6.732933 & 0.502542 & 13.39776 & 0.0000 \\
EFICACIA_POLICIAL & 4.026273 & 0.120260 & 33.47969 & 0.0000 \\
HOMB_JOV & -86.87751 & 2.205930 & -39.38362 & 0.0000 \\
HOMB_JOV_POBRES & -13.39800 & 0.541123 & -24.75962 & 0.0000 \\
HOMB_JOV_URB & 78.87909 & 2.333436 & 33.80384 & 0.0000 \\
PERS_BAJO_LINEA_POBREZA & 11.40754 & 0.583595 & 19.54703 & 0.0000 \\
PLAN_CUADRANTE & 1.770120 & 0.026231 & 67.48080 & 0.0000 \\
POBLACION_URB & -10.86568 & 0.298959 & -36.34502 & 0.0000 \\
APRE_ROBO_FUERZA & -1.400999 & 0.027466 & -51.00916 & 0.0000 \\
CONSUMO_DROGAS_MARIHUANA & 5.960563 & 0.353855 & 16.84467 & 0.0000 \\
DROGAS_DEN_C_100_MIL_HAB & 4.957061 & 0.322881 & 15.35257 & 0.0000 \\
EDAD_PROM_POBLACION & -3.067154 & 0.208115 & -14.73777 & 0.0000 \\
ESCOLARIDAD_PROM_JOV & 10.20440 & 3.609884 & 2.826795 & 0.0368 \\
ESCOLARIDAD_PROM_POBLACIO & 76.88668 & 3.173118 & 24.23064 & 0.0000 \\
N & 0.000584 & $7.04 E-06$ & 82.89213 & 0.0000 \\
MUJ_JEFAS_HOGAR & -118.4241 & 2.865740 & -41.32410 & 0.0000 \\
\hline
\end{tabular}

${ }^{46}$ Jarque Bera $=0,067318$, P-valor $=0,966901$ 
Polít. crim. Vol. 6, No 11 (Julio 2011), Doc. 1, pp. 192 - 208.

[http://www.politicacriminal.cl/Vol_06/n_11/Vol6N11D1.pdf]

\begin{tabular}{ccccc} 
RAZON_DESIGUALDAD_NA & -129.2497 & 6.688960 & -19.32284 & 0.0000 \\
RAZON_DESIGUALDAD_RE & 616.0605 & 32.13348 & 19.17192 & 0.0000 \\
C & & & & \\
\hline
\end{tabular}

Como se observa en la tabla 3 la variable exógena analizada es Robo con Violencia, al estimar el modelo con todas las variables explicativas es relevante mencionar que dos de ellas no presentaron significatividad, por lo cual fueron excluidas del modelo. Las variables referidas corresponden a: hombres jóvenes pobres urbanos y aprehendidos por drogas. En cambio las otras variables explicativas resultan ser significativas individualmente al 99\%, sólo una de estas variables presenta significatividad $95 \%$. Respecto a la significatividad conjunta, se observa que el modelo es significativo también al 99\%. Otro resultado importante de destacar es el coeficiente de determinación $\left(\mathrm{R}^{2}\right)$ donde obtenemos que el porcentaje de explicación del modelo es de aproximadamente un 99,9. Por su parte, el test de normalidad de los residuos según el estadístico de Jarque Bera ${ }^{47}$ indica que los datos presentan un comportamiento normal. Se presenta a continuación el modelo Robo con Violencia en su forma linealizada, considerando los efectos fijos de tiempo seleccionados:

$\mathrm{RV}_{\text {it }}=616.0605+6.732933 *$ DESEMPLEO $+4.026273 *$ EFICACIA_POLICIAL $86.87751 *$ HOMB_JOV $-13.39800 *$ HOMB_JOV_POBRES + $78.87909 *$ HOMB_JOV_URB + $11.40754 *$ POBLACION_POBRE $+1.770120 *$ PLAN_CUADRANTE $-10.86568 *$ POBLACION_URBANA - $1.400999 *$ APRE_ROBO_FUERZA + $5.960563 *$ CONSUMO_DROGAS_MARIHUANA + 4.957061 * DROGAS_DEN_C_100_MIL_HAB $3.067154 *$ EDAD_PROM_POBLACION + $10.20440 *$ ESCOLARIDAD_PROM_JOV + $76.88668 *$ ESCOLARIDAD_PROM_POBLACION + 0.000584 $*$ MUJ_JEFAS_HOGAR $118.4241 *$ RAZON_DESIGUALDAD_NA - 129.2497 * RAZON_DESIGUALDAD_RE + T98 * $(-46.37062)+\mathrm{T}_{00} *(-9.572969)+\mathrm{T}_{06} *(28.76954)$

Donde: $\quad T_{t}=1$ para el período $t(1990, \ldots, 2008)$ y $T_{t}=0$ en caso contrario;

$i=$ Regiones pertenecientes al mapa político antiguo chileno $(1, \ldots, 13)$.

Para el modelo de Hurto, como se ha mencionado el efecto fijo que mejor lo representa es el efecto fijo de empresa, por lo que se consideran trece regiones y un efecto fijo empresa, los resultados de la estimación se presentan en la tabla 4.

Tabla 4: Resultados estimación Hurto

\begin{tabular}{clllc}
\hline Variable & Coefficient & Std. Error & t-Statistic & Prob. \\
\hline EFICACIA_POLICIAL & -5.320313 & 0.250394 & -21.24778 & 0.0000 \\
HOMB_JOV_POBRES & -11.57869 & 1.184127 & -9.778243 & 0.0002 \\
HOMB_JOV_URB_POBRES & -7.406854 & 0.685540 & -10.80441 & 0.0001 \\
HOMB_JOV_URBANOS & -34.44806 & 7.383503 & -4.665543 & 0.0055 \\
PERS_BAJO_LINEA_POBRE & 11.63593 & 1.868041 & 6.228950 & 0.0016 \\
PLAN_CUADRANTE & 1.239305 & 0.145146 & 8.538323 & 0.0004 \\
POBLACIÓN_URBANA & 5.507533 & 1.053043 & 5.230112 & 0.0034 \\
\hline
\end{tabular}

${ }^{47}$ Jarque Bera $=0.641388 ; \mathrm{P}$-valor $=0.725645$ 
DE LA FUENTE, Hanns; MEJÍAS, Claudia; CASTRO, Pía. “Análisis econométrico de los determinantes de la criminalidad en Chile."

\begin{tabular}{cllll}
\hline APREHENDIDOS_ROBO_FUERZA & 1.305315 & 0.085406 & 15.28357 & 0.0000 \\
CONSUMO_DROGAS_MARIHUANA & 8.464732 & 1.475195 & 5.738042 & 0.0023 \\
DROGAS_APRE_C_100_MIL_HA & 0.369433 & 0.053693 & 6.880474 & 0.0010 \\
DROGAS_DEN_C_100_MIL_HA & 14.64760 & 1.476181 & 9.922634 & 0.0002 \\
ESCOLARIDAD_PROM_JOV & 123.5978 & 9.376560 & 13.18158 & 0.0000 \\
ESCOLARIDAD_PROM_POBLACI & -44.05984 & 16.82913 & -2.618070 & 0.0472 \\
MUJ_JEFAS_HOGAR & -0.000519 & $7.61 \mathrm{E}-05$ & -6.827026 & 0.0010 \\
RAZON_DESIGUALDAD_AUT_NA & -137.9740 & 9.471426 & -14.56739 & 0.0000 \\
RAZON_DESIGUALDAD_AUT_RE & -315.1547 & 32.75816 & -9.620648 & 0.0002 \\
C & -449.1310 & 75.10967 & -5.979670 & 0.0019 \\
\hline
\end{tabular}

Respecto de los resultados de la estimación presentados en la tabla 4, cabe destacar que las variables exógenas, Desempleo, Hombres jóvenes y Edad Promedio de la Población, se eliminan del modelo por resultar no significativas. El resto de las variables, resultan significativas al 99\%, no ocurriendo esto con Escolaridad Promedio de la Población la cual representa una significatividad al 95\%. Referente a la significatividad conjunta, se aprecia que el modelo es significativo al 99\%. De acuerdo al coeficiente de determinación $\left(\mathrm{R}^{2}\right)$ se indica que aproximadamente el $99.9 \%$ de la variabilidad en la variable Endógena (Hurto) está siendo explicada por las variables exógenas considerados en el modelo final. Finalmente, respecto a la normalidad de los residuos, estos presentan un comportamiento normal según el estadístico de Jarque Bera. $^{48}$

A continuación se presenta el modelo Hurto en su forma linealizada, considerando los efectos fijos de tiempo seleccionados:

$\operatorname{HURTO}_{i t}=$

$-449.1310-5.320313 *$ EDAD_POBLACION $-11.57869 *$ HOMB_JOV_POBRES -

$7.406854 *$ HOMB_JOV_URB_POBRES - 34.44806 * HOMB_JOV_URB + $11.63593 *$

POBLACION_POBRE + $1.239305 *$ PLAN_CUADRANTE $+5.507533 *$ POBLACION_URB +

$1.305315 *$ APREHENDIDOS_ROBO_FUERZA + $8.464732 *$

CONSUM_DROGAS_MARIHUANA + $0.369433 *$ DROGAS_APRE_C_100_MIL_HA + $14.64760 *$

DROGAS_DENUNCIA_C_100_MIL_HA + $123.5978 *$ ESCOLARIDAD_PROM_JOV - $44.05984 *$

ESCOLARIDAD_PROM_POBLACION $-0.000519 *$ MUJ_JEFAS_HOGAR $-137.9740 *$

RAZON_DESIGUALDAD_NA - 315.1547 * RAZON_DESIGUALDAD_RE + $\mathrm{R}_{1} *(-341.3003)+$ $\mathrm{R}_{2} *(-166.3691)+\mathrm{R}_{3} *(-58.49832)+\mathrm{R}_{4} *(-69.78349)+\mathrm{R}_{5} *(-143.8569)+\mathrm{R}_{6} *$ $(24.70291)+\mathrm{R}_{7} *(240.7147)+\mathrm{R}_{8} *(33.24429)+\mathrm{R}_{9} *(225.3020)+\mathrm{R}_{10} *(219.2590)+$ $\mathrm{R}_{11} *(232.1689)+\mathrm{R}_{12} *(22.12499)+\mathrm{R}_{13} *(-23.86378)$

Donde: $R_{i}=1$ para la región $i(1, \ldots, 13)$ y $R_{i}=0$ en caso contrario;

$t=$ Periodos correspondientes para los años 1990 a 2008.

\footnotetext{
${ }^{48}$ Jarque Bera $=1.235584 ;$ P-valor $=0.539134$
} 
Polít. crim. Vol. 6, No 11 (Julio 2011), Doc. 1, pp. 192 - 208.

[http://www.politicacriminal.cl/Vol_06/n_11/Vol6N11D1.pdf]

\section{Conclusiones}

En la presente investigación se estudia la criminalidad, utilizando como variables de control al Robo con Fuerza, Robo con Violencia y Hurto. Se debe tener presente que los indicadores usados como aproximación a la actividad delictual pueden relacionarse con una serie de elementos, tales como, la cifra negra (delitos no denunciados), el aumento de confianza de la población en relación a las instituciones de justicia, la transparencia en la difusión de la información, entre otros.

Metodológicamente se estudia la criminalidad mediante la estimación de modelos econométricos tradicionales investigándose los factores que motivan a un individuo a cometer delito, para esto se extraen diecinueve factores de la literatura revisada, de los cuales doce se comprueban que presentan gran relevancia en el aumento de la criminalidad en Chile. Así los determinantes de la delincuencia en la sociedad chilena son:

- Dentro de los factores socioeconómicos tenemos que las personas que viven bajo la línea de la pobreza presenta gran motivación para cometer un acto delictivo. Así también ocurre con los individuos que están desempleados y aquellos con bajo nivel educativo, los factores mencionados presentan una gran variación en la tasa de denuncias de delitos.

- Dentro de los factores demográficos se comprueba que la densidad poblacional afecta de gran manera al aumento delictual, es decir, entre mas número de personas vivan en una zona mayor delincuencia se cometerá. Otro factor relevante es la edad, para este estudio se analizan los hombres de 15 a 29 años, que comprueba que los hombres jóvenes son los que más cometen delito. Por su parte, si el promedio de edad en la población aumenta conlleva a que se cometerá menos delitos en la sociedad. Así también destaca el aumento en los robos que puede provocar una inadecuada supervisión parental. El gran aumento en el consumo de drogas en la sociedad ha provocado un aumento considerable en las denuncias por robo, en Chile se ve que en los hogares de las zonas con mayor precariedad existe un fácil acceso a ellas, esto puede tener relación directa con la ausencia parental como se menciona anteriormente.

- Dentro de los factores disuasorios que provocan una disminución en los incentivos que tienen los individuos para cometer delito, es la eficacia policial, es decir la capacidad de la policía para obtener resultados positivos, esto conlleva a que el individuo presente menor motivación en cometer actos ilícitos. Otro factor relevante y que se relaciona con el mencionado anteriormente es el número de aprehendidos, es decir, el individuo como menciona $\mathrm{Ehrlich}^{49}$ visualiza que si la actividad delictiva provoca un aumento en sus utilidades y si el costo (arrestos) de esta actividad es menor, este individuo se siente motivado a cometer delito. Por lo tanto, si aumenta en número de arrestos en la sociedad la tasa de denuncias por robo se verá disminuida.

De acuerdo a los resultados obtenidos de los modelos econométricos se concluye que para el modelo Robo con Fuerza, las variables que determinan la delincuencia corresponden a: Eficacia Policial, Hombres Jóvenes Urbanos Pobres, Hombres Jóvenes Urbanos, Consumo de Drogas (Marihuana), Aprehendidos por Drogas y Mujeres Jefas de Hogar. Por su parte, para el modelo

\footnotetext{
${ }^{49}$ EHRLICH, Participation in Illegitimate Activities, cit. nota ${ }^{\circ}$ 2, p. 2.
} 
DE LA FUENTE, Hanns; MEJÍAS, Claudia; CASTRO, Pía. “Análisis econométrico de los determinantes de la criminalidad en Chile."

Robo con Violencia, las variables que determinan la delincuencia corresponden a: Desempleo, Hombres Jóvenes Urbanos, Personas Bajo la Línea de Pobreza, Aprehendidas por Robo con Fuerza, Consumo de Drogas (Marihuana), Edad Promedio de la Población y Mujeres Jefas de Hogar. Y, para el modelo Hurto, las variables que determinan la delincuencia corresponden a: Eficacia Policial, Personas Bajo la Línea de Pobreza, Población Urbana, Consumo de Drogas (Marihuana) y Escolaridad Promedio de la Población.

De acuerdo a lo antes señalado, los factores relevantes en el aumento de la criminalidad requieren mayor vigilancia de las que han recibido hasta el momento, lo que presume diseñar estrategias sociales que se encarguen eficazmente de estas situaciones, así como políticas impulsoras de empleo para las personas jóvenes en especial en riesgo social, ambos ámbitos en nuestra sociedad se percibe muy débil por parte de la política pública. Otro aspecto es mejorar oportunidades económicas o asegurar una mayor integración para los jóvenes en la sociedad, lo que conlleva a que los jóvenes que se encuentran en riesgo social tengan oportunidad de salir de este riesgo.

Como futuras líneas de investigación se propone profundizar el estudio, considerando las mismas variables, y sólo incorporar a nivel agregado las que pertenecen a políticas públicas, que para el caso de esta investigación, no se ha logrado comprobar la influencia por el escaso periodo en marcha. Además, se recomienda aplicar el estudio tanto a nivel nacional como a nivel comunal, y evaluar las diferencias con la presente investigación.

\section{BIBLIOGRAFÍA}

BANDRÉS E. y DÍEZ-TICIO A., "Delincuencia y acción policial: Un enfoque económico", Revista de Economía Aplicada, Vol. IX, N²7 (2001), pp. 5-33.

BECKER G., "Crime and Punishment: An Economic Approach", Journal of Political Economy, Vol. 76, N² (1986).

BEYER H. y VERGARA R., Delincuencia en Chile: Determinantes y Rol de las Políticas Públicas, mimeo., Pontificia Universidad Católica de Chile, 2006.

BLUMENSTEIN A., COHEN J., ROTH J. y VISHER E., Criminal Careers and "Career Criminals”, Washington DC: National Academy of Sciences, Santiago: Departamento de Economía Universidad de Chile, 1986.

BUONANNO P., Crime, education and peer pressure, Working paper $\mathrm{N}^{\circ}$ 64, Milán: Università degli Studi di Milano, 2003.

, Identifying the effect of education on crime. Evidence from the Italian Regions, Working paper $N^{\circ}$ 65, Milán: Università degli Studi di Milano, 2003.

BUONANNO P. y LEONIDA L., Criminal activity and education: evidence from Italian Regions, Working paper $\mathrm{N}^{\circ}$ 3, Bergamo: Università degli Studi di Bergamo, 2005.

CORMAN H. y MOCAN H., "A Time-Series Analysis of Burglary, Deterrence, and Drug Abuse in New York City", American Economic Review Vol. 90 (2000), pp. 584-604.

EHRLICH I., "Participation in Illegitimate Activities: A Theoretical and Empirical Investigation", Journal of Political Economy, Vol. 81, № 3 (1973).

GLAESER E., SACERDOTE B., y SCHEINKMAN J., "Crime and Social Interactions", Quarterly Journal of Economics Vol. 111 (1996), pp. 507-548.

GREENE W.H., Econometric Analysis Upper Saddle River. New Jersey: Pentice Hall, 2002, $5^{\mathrm{a}}$ Edición. 
Polít. crim. Vol. 6, № 11 (Julio 2011), Doc. 1, pp. 192 - 208.

[http://www.politicacriminal.cl/Vol_06/n_11/Vol6N11D1.pdf]

GUTIÉRREZ M., NÚÑEZ J. y RIVERA J., "Caracterización socioeconómica y espacial de la criminalidad en Chile", Revista Cepal № 98 (2008), pp.165-180.

LEVITT S., "The Effect of Prison Population Size on Crime Rates: Evidence from Prison Overcrowding Litigation”, Quarterly Journal of Economics Vol. CXI (1996), pp. 319-352.

, "Using electoral cycles in Police Hiring to Estimate the Effect of Police on Crime", American Economic Review, Vol. 87, №3 (1997).

LOCHNER L. y MORETTI E., The Effect of Education on Crime: Evidence from Prison Inmates, Arrests, and Self-Reports, NBER Working paper N 8605, 2001.

MACHIN S., y MEGHIR C., "Crime and Economic Incentives", Journal of Human Resources, Vol. 39, N 4 (2004), pp. 958-979.

MARVELL T., y MOODY, C., "Age Structure and Crime Rates: The Conflicting Evidence", Journal of Quantitative Criminology, Vol. 7, N³ (1991), pp. 237-273.

"Prison population growth and crime reduction", Journal of Quantitative Criminology, Vol. 10, N 1 (1994), pp. 109-140.

PÉREZ C., Econometría de las series temporales, Pearson- Prentice Hall.

RODRÍGUEZ A., "Los determinantes socio-económicos del delito en España", Revista Española de Investigación Criminológica $\mathrm{N}^{\circ} 1$ (2003).

RUIZ P, CEA M, RODRÍGUEZ C, MATUS J.P., "Determinantes de la criminalidad: Análisis de Resultados", Política Criminal N³ (2007), pp. 1-80.

WILSON J. y HERRENSTEIN R., Crime and Human Nature: The Definitive Study of the Causes of Crime, New York: Simon \& Schuster, 1985. 